\title{
Testing scenarios on geological models: local interface insertion in a 2D mesh and its impact on seismic wave simulation
}

\author{
C. Legentil ${ }^{\dagger, *}$, J. Pellerin ${ }^{\ddagger}$, P. Cupillard ${ }^{\dagger}$ and G. Caumon ${ }^{\dagger}$ \\ $\dagger$ GeoRessources \\ Université de Lorraine - CNRS \\ 54000 Nancy, France \\ $\ddagger$ Total \\ 91120 Palaiseau, France
}

\begin{abstract}
Forecasting the physical behavior of the subsurface is a challenging task. Available data include seismic wave propagation recordings that are interpreted to build subsurface multi-material models. Due to this interpretation, the geometry and the connectivity of the interfaces separating geological materials are uncertain. In this work, we consider a relatively simple case of fluid monitoring in a subsurface gas reservoir. As seismic wave velocities of porous rocks vary depending on fluid saturation, our objective is to quantitatively evaluate the impact of the water/gas contact depth on elastic wave propagation.

To efficiently test different contact depth scenarios and assess their impact on wave propagation, we propose to locally modify a 2D geological model and run time-dependent elastic simulations (Fig. 1). The input model is a triangulated surface conforming to geological structures and representing physical properties. The $2 \mathrm{D}$ meshed model is locally updated, meaning that only a given region is modified and that the other parts of the mesh remain identical. To create several models by modifying only the reservoir layer, we insert a water/gas contact defined by a level-set at several depths with MMG [1]. During the insertion, specific care is taken to maintain the conformity of the output mesh. As compared to global remeshing, the local modification reduces the cost of recomputing physical properties over the updated mesh [2]. The level-set formulation is interesting for generalizations to more complex interfaces. We run the numerical simulations with Hou10ni2D code [3], which is based on a Discontinuous Galerkin method. The simulation results are compared with the reference by computing the L2-norm at a set of seismic receivers on the Earth surface. Our results on a gas reservoir show a consistent behavior: we observe a correlation between the depth difference and L2-norm, the larger the distance from the reference depth contact, the higher the L2-norm. This approach could therefore be integrated into an inversion loop to determine the position of the fluid contact and reduce uncertainties in the reservoir model from a few seismic sensors. More generally, the local insertion of an implicit interface into an existing mesh opens interesting perspectives to address other types of geological uncertainties (e.g., the simulations of undetected fractures or faults, or the management of stratigraphic uncertainties).
\end{abstract}




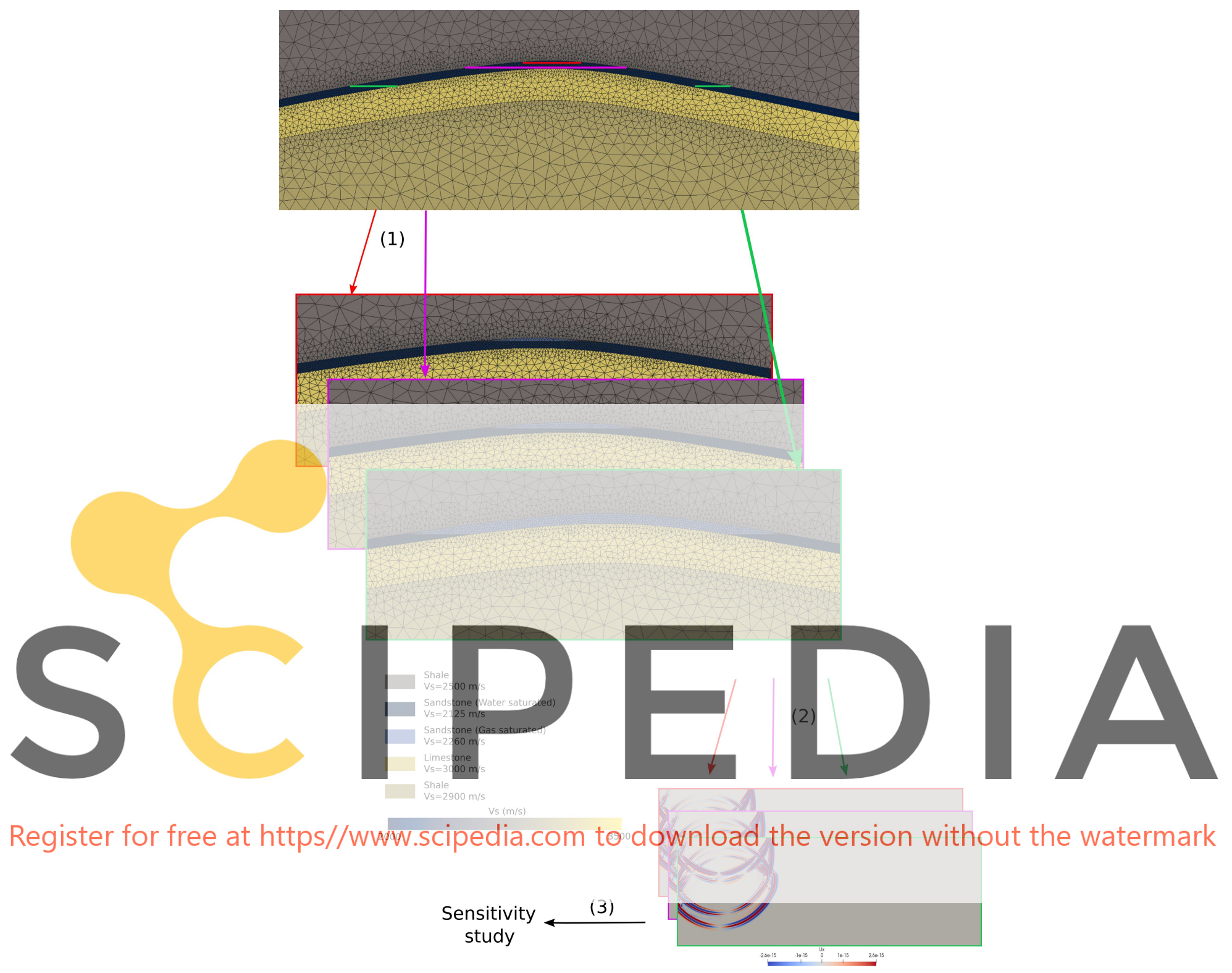

Figure 1: (1) From several level-sets defining the possible interface positions between water-saturated sandstone and gas-saturated sandstone, we use MMG [1] to locally remesh the dark blue layer and (2) the Hou10ni2D software [3] to simulate wave propagation and (3) compare the simulation results.

\section{REFERENCES}

[1] Dapogny, C., Dobrzynski, C., and Frey, P. (2014). Three-dimensional adaptive domain remeshing, implicit domain meshing, and applications to free and moving boundary problems. Journal of Computational Physics, 262:358-378.

[2] Suter, E., Cayeux, E., Friis, H. A., Kårstad, T., Escalona, A., and Vefring, E. (2017). A Novel Method for Locally Updating an Earth Model While Geosteering. International Journal of Geosciences, 08(02):237-264.

[3] Barucq, H., Djellouli, R., and Estecahandy, E. (2014). Efficient dg-like formulation equipped with curved boundary edges for solving elasto-acoustic scattering problems. International Journal for Numerical Methods in Engineering, 98(10):747-780. 